\title{
Rehabilitación de maxilar atrófico con implantes cigomáticos. Presentación de un caso y revisión de la literatura
}

\author{
Rehabilitation of atrophic maxilla with zygomatic implants. \\ Case report and literature review
}

\author{
Juan Peña Lares,* Osmar Adán Cárcamo Idiáquez, \\ Eliot Samuel Velázquez Varela, ${ }^{\S}$ Israel Vivanco Pérez, "Adolfo Navarro Zárate," \\ Martín Gilberto Flores Ávila,** Gerardo Romero Jasso
}

\section{RESUMEN}

Introducción: La rehabilitación estética y funcional en pacientes con secuelas por resección de neoplasias, traumatismos o malformaciones congénitas de la región maxilofacial, así como pacientes con maxilares atróficos por otras causas, es un reto para cirujanos maxilofaciales y rehabilitadores orales debido a la limitada cantidad y

\section{ABSTRACT}

Introduction: Aesthetic and functional rehabilitation in patients with sequelae due to resection of neoplasms, trauma or congenital malformations of the maxillofacial region, as well as patients with atrophic jaws due to other causes, is a challenge for maxillofacial surgeons and oral rehabilitators due to the limited quantity and quality of

* Cirujano maxilofacial en Hospital Christus Muguerza UPAEP. Puebla, Puebla, México. Profesor colaborador del postgrado de Cirugía Maxilofacial, Hospital Universitario de Puebla. Benemérita Universidad Autónoma de Puebla. Puebla, Puebla, México.

${ }^{\ddagger}$ Médico residente de cuarto año Cirugía Maxilofacial, Hospital Universitario de Puebla. Benemérita Universidad Autónoma de Puebla. Puebla, Puebla, México. $\S$ Cirujano maxilofacial, práctica privada en consultorio CECMI. León, Guanajuato, México.

" Cirujano maxilofacial, Jefe de Servicio y Profesor Titular del postgrado de Cirugía Maxilofacial, Hospital Universitario de Puebla. Benemérita Universidad Autónoma de Puebla. Puebla, Puebla, México.

" Cirujano maxilofacial, médico adscrito y profesor adjunto del postgrado de Cirugía Maxilofacial, Hospital Universitario de Puebla. Benemérita Universidad Autónoma de Puebla. Puebla, Puebla, México.

** Cirujano maxilofacial, médico adscrito al Servicio de Cirugía Maxilofacial, Hospital Regional "Lic. Adolfo López Mateos», ISSSTE. Ciudad de México, México. 执 Cirujano maxilofacial, práctica privada, Ciudad de México, México.

Correspondencia: Juan Peña Lares

Dirección: Av. 5 Pte. 715, Col. Centro, 72000, Puebla, Puebla, México, Tel: 01222 229-8100.

E-mail: maxilofacial@penalares.com

Citar como: Peña LJ, Cárcamo IOA, Velázquez VES, Vivanco PI, Navarro ZA, Flores ÁMG et al. Rehabilitación de maxilar atrófico con implantes cigomáticos. Presentación de un caso y revisión de la literatura. Rev Mex Cir Bucal Maxilofac. 2021;17 (2): 96-108. https://dx.doi.org/10.35366/102432 
calidad de hueso remanente en el proceso alveolar maxilar residual. Los implantes cigomáticos son una opción efectiva y definitiva en el manejo de este tipo de pacientes y su objetivo será proporcionar una retención en la región maxilar posterior en un paciente que no es candidato para la colocación de implantes convencionales debido a la atrofia maxilar, lo que le permitirá una rehabilitación integral con menor grado de morbilidad y en menos tiempo, evitando así procedimientos de injertos óseos. Caso clínico: Se presenta el caso de una paciente de 61 años de edad con diagnóstico de atrofia maxilar severa en zonas 1-2-3 según Bedrossian, a quien se le realiza protocolo de rehabilitación con cuatro implantes cigomáticos con carga inmediata. Actualmente la paciente se encuentra asintomática y en espera de prótesis definitiva. Conclusión: Los implantes cigomáticos son un recurso que proporciona una solución predecible y rápida al problema de déficit óseo como resultado de una atrofia maxilar o maxilectomía, el implante cigomático no conlleva mayor complejidad protésica para el paciente y ha mostrado resultados clínicos superiores en comparación con el injerto óseo, por lo que representan un nuevo «estándar de oro» en cuanto al tratamiento de los huesos maxilares comprometidos.

Palabras clave: Atrofia maxilar, rehabilitación protésica, implantes cigomáticos, carga inmediata. bone remaining in the residual maxillary alveolar process. Zygomatic implants are an effective and definitive option in the management of this type of patients and their objective is to provide retention in the posterior maxillary region, in a patient who is not a candidate for the placement of conventional implants due to maxillary atrophy, which will allow an integral rehabilitation with less morbidity and in less time, thus avoiding bone grafting procedures. Clinical case: We present the case of a 61-year-old female patient with a diagnosis of severe maxillary atrophy in zones 1-2-3 according to Bedrossian, who underwent a rehabilitation protocol with 4 zygomatic implants with immediate loading. Currently the patient is asymptomatic and awaiting definitive prosthesis. Conclusion: Zygomatic implants are a resource that provides a predictable and rapid solution to the problem of bone deficit as a result of maxillary atrophy or maxillectomy, the zygomatic implant does not entail greater prosthetic complexity for the patient and has shown superior clinical results compared to bone grafting, thus representing a new gold-standard in the treatment of compromised maxillary bones.

Keywords: Maxillary atrophy, prosthetic rehabilitation, zygomatic implants, immediate loading.

\section{INTRODUCCIÓN}

Los pacientes con secuelas por traumatismos, resección de neoplasias o malformaciones congénitas de la región maxilofacial, así como los pacientes con atrofia maxilar severa por diferentes etiologías (edad avanzada, pérdida dental no traumática, utilización de prótesis totales por largos periodos, neumatización de senos maxilares) representan un reto para la rehabilitación estética y funcional con implantes dentales convencionales debido a la limitada cantidad y calidad de hueso remanente. El implante cigomático fue introducido por Branemark para la rehabilitación protésica en pacientes con defectos maxilares extensos secundarios a resecciones tumorales, traumatismos y defectos congénitos. Branemark e Higuchi en 1999 reportaron un paciente con secuelas postmaxilectomía rehabilitado con un implante cigomático, a partir de ese reporte numerosos estudios se han realizado demostrando que los implantes cigomáticos son una opción efectiva para la rehabilitación de los casos con déficit óseo maxilar. El objetivo del implante cigomático es proporcionar una retención en la región maxilar posterior en un paciente que no es candidato para la colocación de implantes convencionales en esa zona, la utilización del hueso cigomático como un sitio de anclaje para implantes reduce la morbilidad quirúrgica y simplifica el tratamiento de rehabilitación en este tipo de pacientes..$^{1-3} \mathrm{El}$ hueso cigomático es un hueso par localizado en la región antero-lateral del esqueleto facial, el cual forma parte del piso y pared lateral orbitaria así como del techo del seno maxilar. Las dimensiones del cuerpo del hueso cigomático a nivel medio-lateral son de $7.6 \mathrm{~mm}$ en hombres y $8 \mathrm{~mm}$ en mujeres. A nivel anteroposterior $25.4 \mathrm{~mm}$ en hombres y $24.9 \mathrm{~mm}$ en mujeres y su espesor cortical lateral es de $1.75 \mathrm{~mm}$ en hombres y $1.71 \mathrm{~mm}$ en mujeres. Su función es transmitir las fuerzas masticatorias, éste no sufre cambios morfológicos aun en presencia de atrofia maxilar severa. La estabilidad inicial del implante cigomático se deriva de la retención mecánica entre la superficie del implante y el tejido óseo. Nkenke y colaboradores concluyeron que el hueso trabecular del cigomático no era favorable para la colocación de implantes y sugirieron que el éxito observado con los implantes cigomáticos es probablemente el resultado de la participación de cuatro corticales (cortical palatina, cortical del piso del seno maxilar en la porción crestal del implante y las corticales óseas cigomáticas en el ápice del implante). ${ }^{4-6}$ 
El implante cigomático tiene una longitud de $30-55 \mathrm{~mm}$, un diámetro desde $4 \mathrm{~mm}$ a nivel apical hasta $5 \mathrm{~mm}$ a nivel crestal. La integración del implante se produce en el cuerpo del hueso cigomático (longitud integrada de 15 a $20 \mathrm{~mm}$ ). La trayectoria del implante recorre la cresta del contrafuerte cigomático-maxilar y su cabeza hexagonal externa aparece en el área correspondiente al primer molar y segundo premolar. La porción protésica o cabeza del implante se encuentra angulada a 45 grados (Nobelbiocare, Zürich, Suiza) o 55 grados (Southern Implants, Irene, Sudáfrica) para orientarlo lo más paralelo posible al plano oclusal. Los implantes son autorroscables y pueden ser de superficie roscada y texturizada, roscada no texturizada o lisos con roscas apicales. La longitud del implante hace inapropiada su rehabilitación sin la utilización de un conector transversal rígido, el cual permitirá estabilizarlo así como distribuir y compartir la carga oclusal. ${ }^{7,8}$

El estudio auxiliar para el diagnóstico y planificación quirúrgica es la tomografía computarizada (TC) helicoidal, pero el bajo costo y los niveles muy bajos de radiación asociados con la TC de haz cónico hacen que esta última sea preferible. La TC es crucial para evaluar el estado sinusal, la cantidad de hueso cigomático y alveolar residual, la trayectoria y angulación, el sitio de colocación y de emergencia del implante así como la relación del cuerpo del implante con el seno maxilar y su pared lateral. ${ }^{9,10}$

Las ventajas de los implantes cigomáticos en un paciente con atrofia maxilar incluyen la capacidad de rehabilitación con menor morbilidad y en menos tiempo, evitando así procedimientos de injerto óseo. En el protocolo original el procedimiento se realiza en dos etapas, para lo cual después de seis meses de oseointegración se retira la prótesis provisional y se comprueba la estabilidad de los implantes. La oseointegración se confirma al no existir movilidad de los implantes con la técnica de par reverso (10 Ncm), igualmente si no presenta sensibilidad durante la percusión. Una vez determinada la oseointegración el paciente está listo para la fabricación de una prótesis perfilada acrílica o una prótesis perfilada de metalacrílico usando dientes de resina. Sin embargo, el protocolo original fue reemplazado por uno de carga inmediata con buenos resultados a largo plazo. El criterio para la carga inmediata es tener un mínimo de $40 \mathrm{Ncm}$ de torque de inserción de los implantes cigomáticos. ${ }^{11,12}$

Las desventajas del procedimiento quirúrgico incluyen una disección más extensa, la experiencia del operador, conocimiento de la anatomía sinusal, cigomática y orbitaria así como la necesidad de anestesia general o local con sedación para su realización.

Los implantes cigomáticos están indicados en los pacientes con defectos óseos maxilares, ya sea por secuelas de traumatismos, resección de neoplasias, malformaciones congénitas así como pacientes con atrofia maxilar severa. La principal indicación es pacientes completamente desdentados con neumatización significativa del seno maxilar y atrofia severa del reborde alveolar que requieran soporte maxilar posterior para su rehabilitación integral. También se indican para el tratamiento de pacientes en los que han fracasado los implantes convencionales y/o procedimientos de injertos óseos. Los pacientes con atrofia maxilar son a menudo de edad avanzada y pueden tener comorbilidades que los hacen mejores candidatos para una sola etapa quirúrgica y de rehabilitación. ${ }^{10}$ Bedrossian y colaboradore ${ }^{13}$ clasificaron el maxilar en tres zonas de atrofia potencial, las cuales pueden ser unilaterales o bilaterales, lo que nos permite entender qué pacientes son candidatos para ser rehabilitados con implantes cigomáticos. (Tabla 1 y Figura 1). Las contraindicaciones se pueden clasificar en definitivas y relativas. Dentro de las primeras se incluyen patología sinusal aguda, limitación de apertura oral, contraindicación médica que no permita sedación, anestesia general o cirugía. En las relativas se encuentran: patología sinusal crónica (rinosinusitis alérgica, pólipos nasales, sinusitis fúngica crónica, desviación septal, sinusitis crónica) así como la presencia de dientes mandibulares en región de canino-premolar.

Según la clasificación propuesta por Bedrossian, se recomiendan las siguientes opciones de tratamiento (Tabla 2).

La técnica original descrita por Branemark ${ }^{7}$ consiste en realizar una incisión crestal completa hasta la línea media anterior junto con incisiones posteriores con relajantes posterolaterales bilaterales. Esta técnica implica una exposición subperióstica completa de la cara lateral del hueso cigomático, un retractor se coloca en la escotadura cigomática justo en la unión del arco cigomático y el borde orbitario lateral, se crea una ventana ósea de $10 \mathrm{~mm}$ de ancho en la parte lateral del seno maxilar siguiendo la trayectoria deseada del implante cigomático. La membrana sinusal se diseca cuidadosamente para permitir el paso del implante justo dentro de la pared del seno maxilar 
Tabla 1: Clasificación Bedrossian. ${ }^{13}$

\begin{tabular}{cl}
\hline Zona & Región \\
\hline 3 & Molar \\
2 & Premolares \\
1 & Incisivos y caninos \\
\hline
\end{tabular}

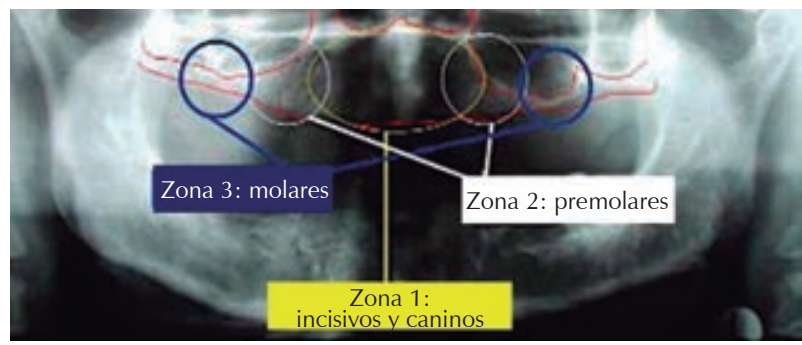

Figura 1: Ortopantomografía del paciente.

Tabla 2: Opciones de tratamiento.

\begin{tabular}{ll}
\hline Atrofia maxilar & Tratamiento \\
\hline Sin atrofia & $\begin{array}{l}\text { Implantes convencionales } \\
\text { All on four, elevación de seno e } \\
\text { implantes convencionales }\end{array}$ \\
Atrofia en zona 3 & $\begin{array}{l}\text { 2-4 implantes convencionales } \\
\text { con dos implantes cigomáticos }\end{array}$ \\
Atrofia en zona 1, 2,3 & Cuatro implantes cigomáticos \\
\hline
\end{tabular}

mientras se dirige hacia el cuerpo del malar. Con abundante irrigación se utiliza una fresa redonda para crear una guía y luego el drill inicial de 2.9 $\mathrm{mm}$ de diámetro hasta que la punta del mismo sale cerca del arco cigomático, posteriormente se utiliza un drill piloto de transición $(2.9-3.5 \mathrm{~mm})$ y por último, el drill de $3.5 \mathrm{~mm}$. Una vez completada la preparación se utiliza el medidor de profundidad para determinar la longitud del implante a utilizar. El implante es llevado a boca y colocado en su preparación. El dispositivo de transferencia se retira para exponer la orientación de 45 grados de la plataforma del implante. Este abordaje da lugar a que la plataforma se extienda sobre el palatino de la cresta alveolar requiriendo de este modo los pilares angulados (UCLA) para evitar la sobrecarga de la prótesis a lo largo del aspecto palatino. La conexión del pilar suele hacerse después de un periodo de cicatrización de seis meses usando pilares estándar o recto/angulado de Branemark multi-unit. $^{\text {? }}$

La técnica propuesta por Stella y Warner ${ }^{14}$ permite la colocación del implante en o muy cerca de la cresta alveolar, no es necesaria la disección completa de la cara lateral del hueso cigomático y

Tabla 3: Clasificación Zaga.

\begin{tabular}{|c|c|}
\hline Zaga & \\
\hline 0 & $\begin{array}{l}\text { La pared maxilar anterior es muy plana. La primera osteotomía se coloca sobre la cresta alveolar residual. El cuerpo del } \\
\text { implante alcanza el hueso cigomático siguiendo una trayectoria intrasinusual }\end{array}$ \\
\hline 1 & $\begin{array}{l}\text { La pared maxilar anterior ligeramente cóncava y la necesidad de colocar la cabeza del implante en el sitio protésico } \\
\text { correcto llevan a que la osteotomía perfore la pared maxilar. La mayor parte del cuerpo del implante permanecerá } \\
\text { dentro de los límites maxilares }\end{array}$ \\
\hline 2 & $\begin{array}{l}\text { En presencia de una pared maxilar más cóncava, la colocación ideal de la cabeza del implante obliga a que la mayor } \\
\text { parte del cuerpo del implante sea colocado de forma extrasinusal sin dejar espacio entre la superficie del implante y el } \\
\text { hueso maxilar anterior }\end{array}$ \\
\hline 3 & $\begin{array}{l}\text { En un maxilar muy cóncavo la primera osteotomía realizada desde la cresta alveolar palatina emerge por la cara anterior } \\
\text { maxilar hasta alcanzar el cigomático en una posición más craneal. La parte media del implante no toca el hueso maxilar }\end{array}$ \\
\hline 4 & $\begin{array}{l}\text { En un maxilar muy atrófico, la colocación óptima del implante debe ser extramaxilar sin perforar la cresta alveolar } \\
\text { residual muy delgada }\end{array}$ \\
\hline
\end{tabular}
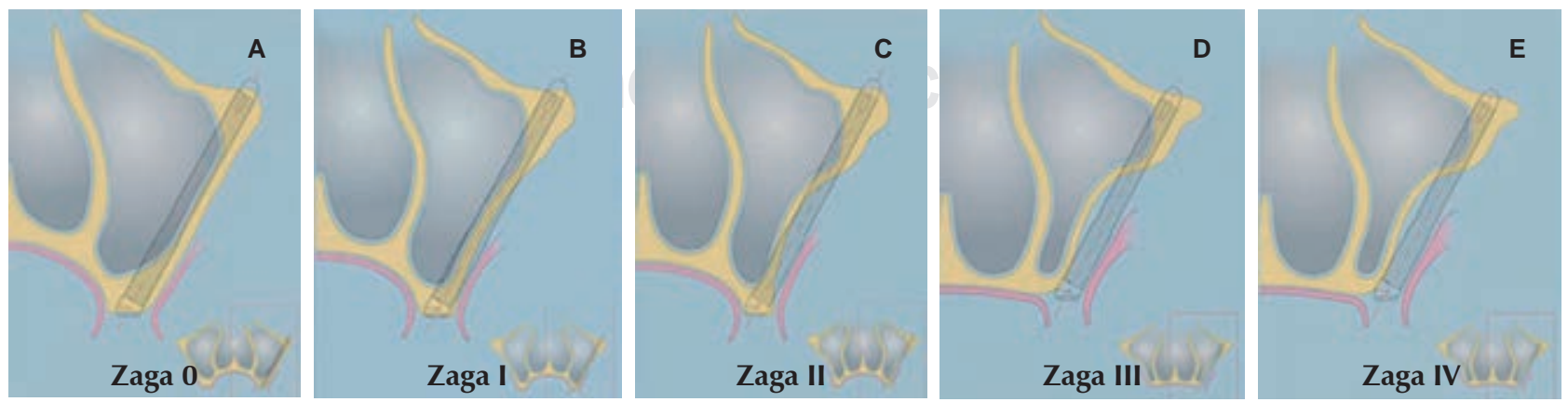

Figura 2: Clasificación Zaga. 
el retractor de escotadura no se utiliza. El agujero de emergencia se perfora en la cresta del alveolo al pie del contrafuerte, una ranura oblicua vertical en la cresta del pilar se crea con una fresa de fisura y termina cefálicamente en el hueso más grueso del cuerpo cigomático. En lugar de un agujero de acceso como en la técnica estándar, la ranura se ensancha hasta convertirse en una superficie plana, la cual es esculpida en el hueso más grueso en la base del cuerpo cigomático. En la superficie plana se realiza un agujero con fresa redonda. Se coloca un dedo sobre la escotadura cigomática en lugar de verla directamente con el retractor. La perforación, el paso del implante y la alineación proceden como en la técnica convencional, excepto que el drill y el implante pasan a través de la ranura en el pilar con el implante situado a menudo parcialmente fuera del seno maxilar. El implante entonces se encuentra en o cerca de la cresta alveolar. ${ }^{14}$ Otra modificación de la técnica original fue descrita por Boyes-Varley y colaboradores, ${ }^{15}$ estos autores abogan por el uso de una guía quirúrgica específica y la modificación del diseño del implante para aumentar la angulación de la plataforma a 55 grados en lugar de los 45 grados, su técnica resulta en la colocación del dispositivo en la cresta del alvéolo con una angulación del implante que se aproxima más a la vertical. Chow y colaboradores proponen un protocolo de rehabilitación con implantes cigomáticos usando planeación virtual con utilización de guías quirúrgicas, incisiones mínimas y carga inmediata. También se ha informado de la navegación intraoperatoria asistida por computadora para ayudar en la colocación de implantes cigomáticos. ${ }^{15}$

En pacientes con concavidades bucales pronunciadas en la parte lateral del seno maxilar, el uso
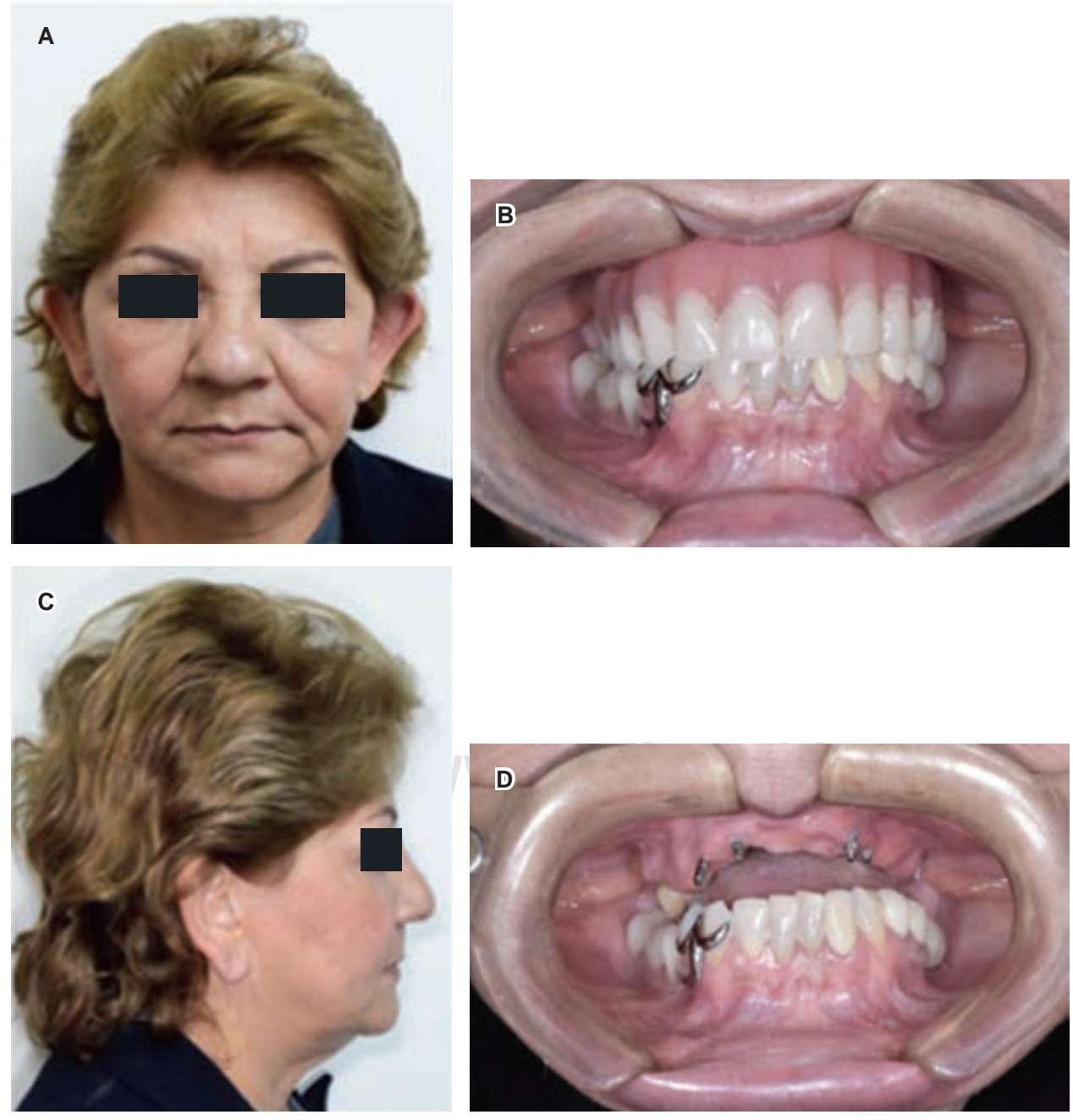

Figura 3:

A) Foto frontal. B) Foto intraoral con su prótesis. C) Foto lateral. D) Foto intraoral sin prótesis. 
de la técnica original con un trayecto intrasinusal da lugar a una emergencia palatina excesiva de la cabeza del implante, esto resulta en un puente dental voluminoso en el aspecto palatino que conduce a un malestar en el paciente con problemas de higiene y habla. ${ }^{17}$ Con el fin de utilizar una aproximación anatómica y protésica, la técnica original se ha modificado permitiendo una trayectoria extrasinusal para los implantes cigomáticos, centrándose en las diferencias anatómicas de cada paciente. La preparación del sitio del implante se rige por la anatomía del área y no es necesario abrir ninguna ventana o ranura inicial en la pared lateral del seno maxilar. De este modo, dependiendo de la relación entre el contrafuerte cigomático y el punto de inicio intraoral del implante cigomático, la trayectoria del cuerpo del implante variará de ser totalmente intrasinusal a ser totalmente extrasinusal. Este enfoque promueve la colocación del implante cigomático según la anatomía del paciente partiendo de un sistema de clasificación que comprende cinco grupos (ZAGA 0-IV). La colocación del implante cigomático sigue los principios ZAGA y optimiza el soporte proporcionado por el hueso, incluso a nivel de la pared maxilar que es crucial en un paciente que sufre de atrofia ósea extrema. El implante por sí mismo sella la osteotomía de la pared sinusal, lo que minimiza el riesgo de la contaminación sinusal ${ }^{18}$ (Tabla 3 y Figura 2).

La colocación de implantes cigomáticos es un procedimiento de cirugía ambulatoria o de corta estancia dependiendo del tipo de paciente. El manejo médico con antibióticos, analgésicos, antiinflamatorios, descongestionantes nasales, entre otros, está reservado a criterio del clínico. Se debe aconsejar reposo relativo, dieta blanda, evitar acciones que aumenten la presión intranasal e intrasinusal. Se recomiendan citas de seguimiento una vez por semana en los primeros seis meses. Dentro de las complicaciones que se pueden presentar se encuentran: dolor, edema, equimosis, hematoma, inflamación, infección, patología sinusal, necrosis ósea, lesiones nerviosas (parestesia, anestesia, paresia), lesión ocular u orbitaria, lesión vascular (arteria maxilar interna, plexo venoso pterigoideo en la fosa pterigopalatina/infratemporal), pérdida del implante, entre otros. ${ }^{19}$

\section{CASO CLÍNICO}

Se presenta el caso de una paciente de 61 años de edad con antecedente de resección mamaria por tumoración benigna, tabaquismo activo desde los 10 años de edad, alérgica a penicilina y sulfas, actualmente en tratamiento con benzodiacepinas para control de depresión senil. Acude a valoración al Servicio de Cirugía Maxilofacial del Hospital Christus Muguerza UPAEP en Puebla, Puebla, México buscando una rehabilitación integral. La paciente refiere movilidad en prótesis maxilar, lo que le condiciona dificultad para la masticación y el desarrollo de sus actividades diarias. A la exploración clínica la paciente presenta una prótesis total maxilar desajustada, al retiro de la misma se observa la presencia de cuatro implantes transicionales endoóseos, los cuales se encuentran asintomáticos, pero con evidente movilidad. El reborde alveolar maxilar se encuentra con atrofia severa en sector anterior y posterior, presenta órgano dentario número 16 con movilidad grado III. A nivel mandibular se encuentra parcialmente desdentada con presencia de prótesis parcial removible en sector posterior bilateral, en el sector anterior con presencia de órganos dentarios en adecuadas condiciones presenta también un implante transicional endoóseo en región posterior derecha (Figura 1).

La exploración clínica se complementa con una ortopantomografía y una tomografía computarizada de haz de cono, las cuales revelan atrofia maxilar severa en zonas 1, 2 y 3 según la clasificación de Bedrossian. Presencia de cuatro implantes transicionales con pérdida ósea periimplantar severa, senos maxilares neumatizados y sin evidencia de patologías paranasales y óseas asociadas (Figura 2).

Con base en la historia clínica, exploración física, radiográfica y tomográfica, así como las necesidades funcionales y sociales de la paciente, se propone una rehabilitación con carga inmediata sobre cuatro implantes cigomáticos. La paciente acepta el plan de tratamiento, por lo que se somete al protocolo preoperatorio, no encontrándose contraindicación para la realización del procedimiento quirúrgico ni anestésico.

\section{MATERIAL Y MÉTODOS}

Se realiza cirugía virtual con apoyo del programa Mimics Research 17.0 Materialise Company, donde se retiran implantes transicionales endoóseos, se extrae órgano dentario 16 y se colocan cuatro implantes cigomáticos permitiendo una aproximación de la posición quirúrgica ideal respetando estructuras anatómicas adyacentes y diseñando 

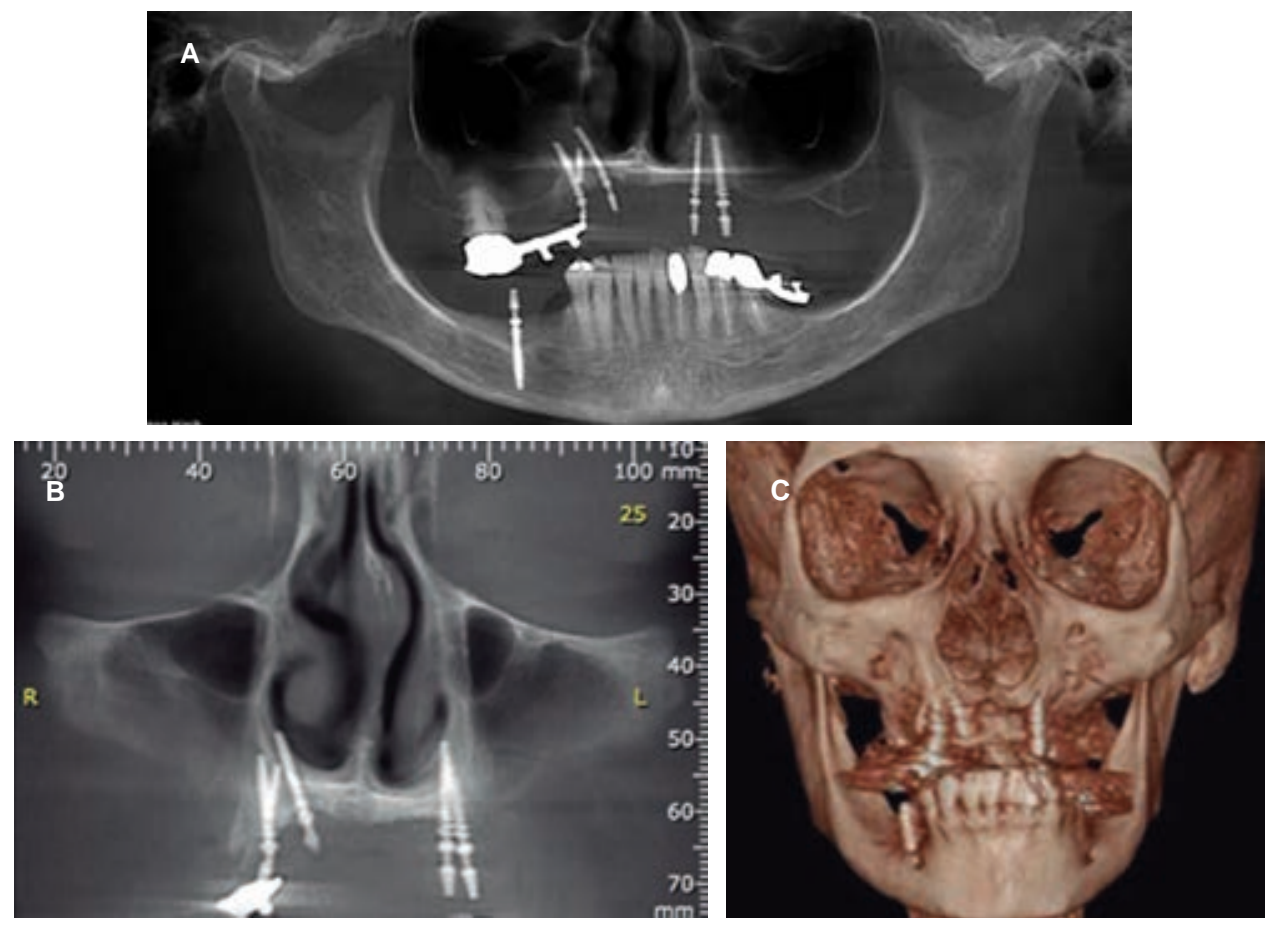

Figura 4:

A) Ortopantomografía inicial. B) Corte coronal donde se observan los implantes transicionales. C) Reconstrucción volumétrica.
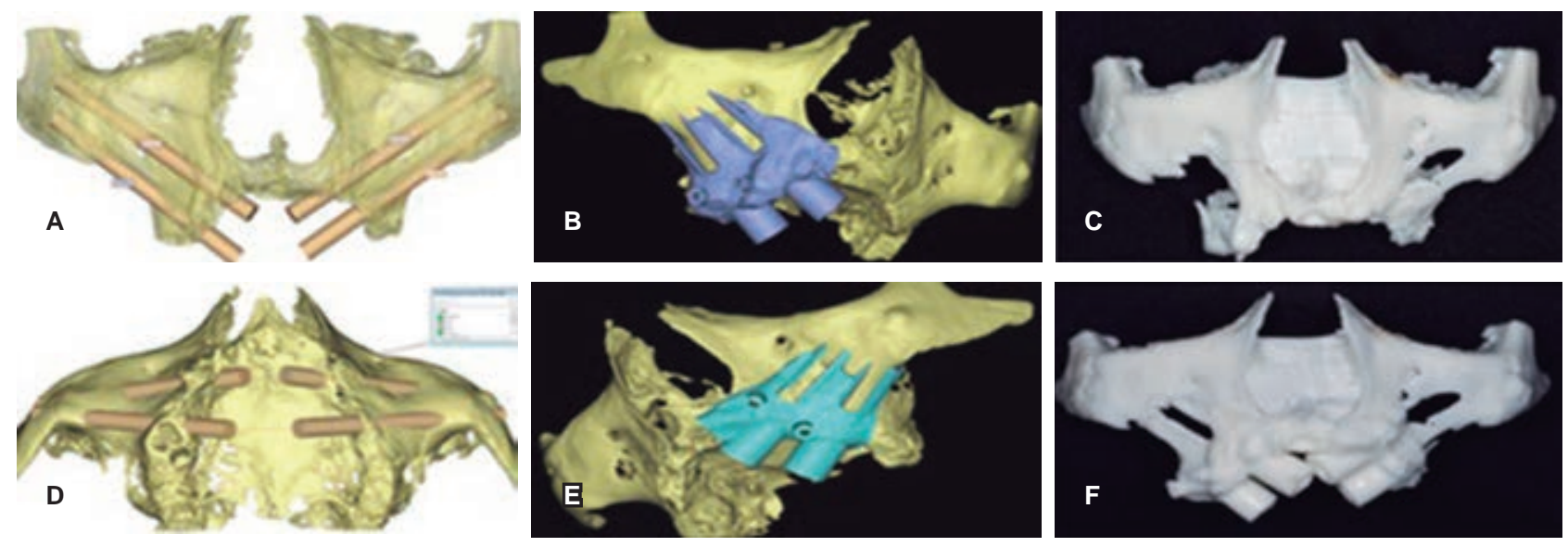

Figura 5: A-F) Planeación digital.

una guía quirúrgica que nos permita determinar el perfil de emergencia de los implantes cigomáticos. Una vez realizada la planeación virtual se fábrica un modelo estereolitográfico así como las guías quirúrgicas que nos orientaran durante el evento quirúrgico (Figura 3).

\section{Técnica quirúrgica}

La paciente es llevada a la sala de operaciones y bajo anestesia general balanceada con intu- bación nasotraqueal, previa asepsia y antisepsia se coloca taponamiento faríngeo, se inicia evento quirúrgico con infiltración de lidocaína con epinefrina al 2\%-1:100,000 para hemostasia regional e hidrodisección de la región anterior y crestal maxilar. Se realiza extracción de O.D. 16 así como retiro de implantes transicionales. Se hace incisión con bisturí No. 15 a nivel crestal maxilar continuándose con una incisión relajante posterolateral de manera bilateral, se diseca un colgajo de espesor total paracrestal palatino, 
el cual se sutura temporalmente con el contralateral con seda 3-0. Posteriormente se diseca un colgajo de espesor total exponiendo la cara anterior maxilar, pilar maxilomalar y borde inferior del arco cigomático, enseguida con fresa de bola diamantada para baja velocidad y abundante
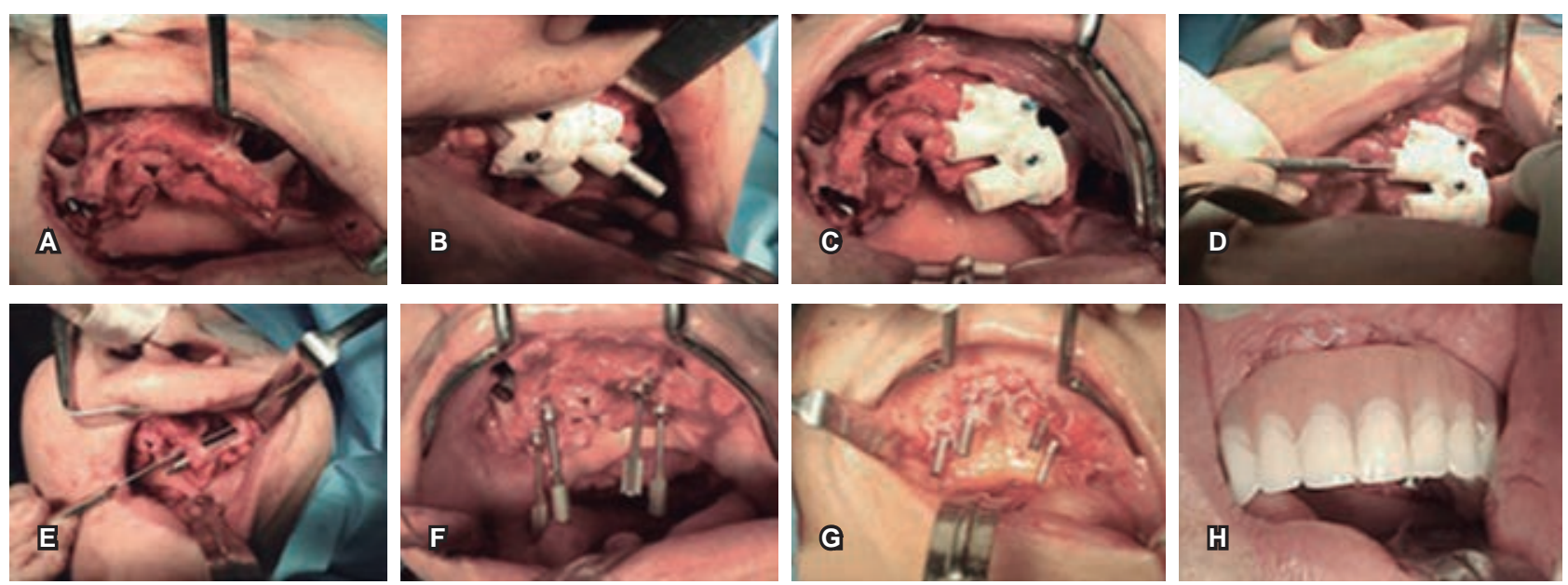

Figura 6: Procedimiento quirúrgico.
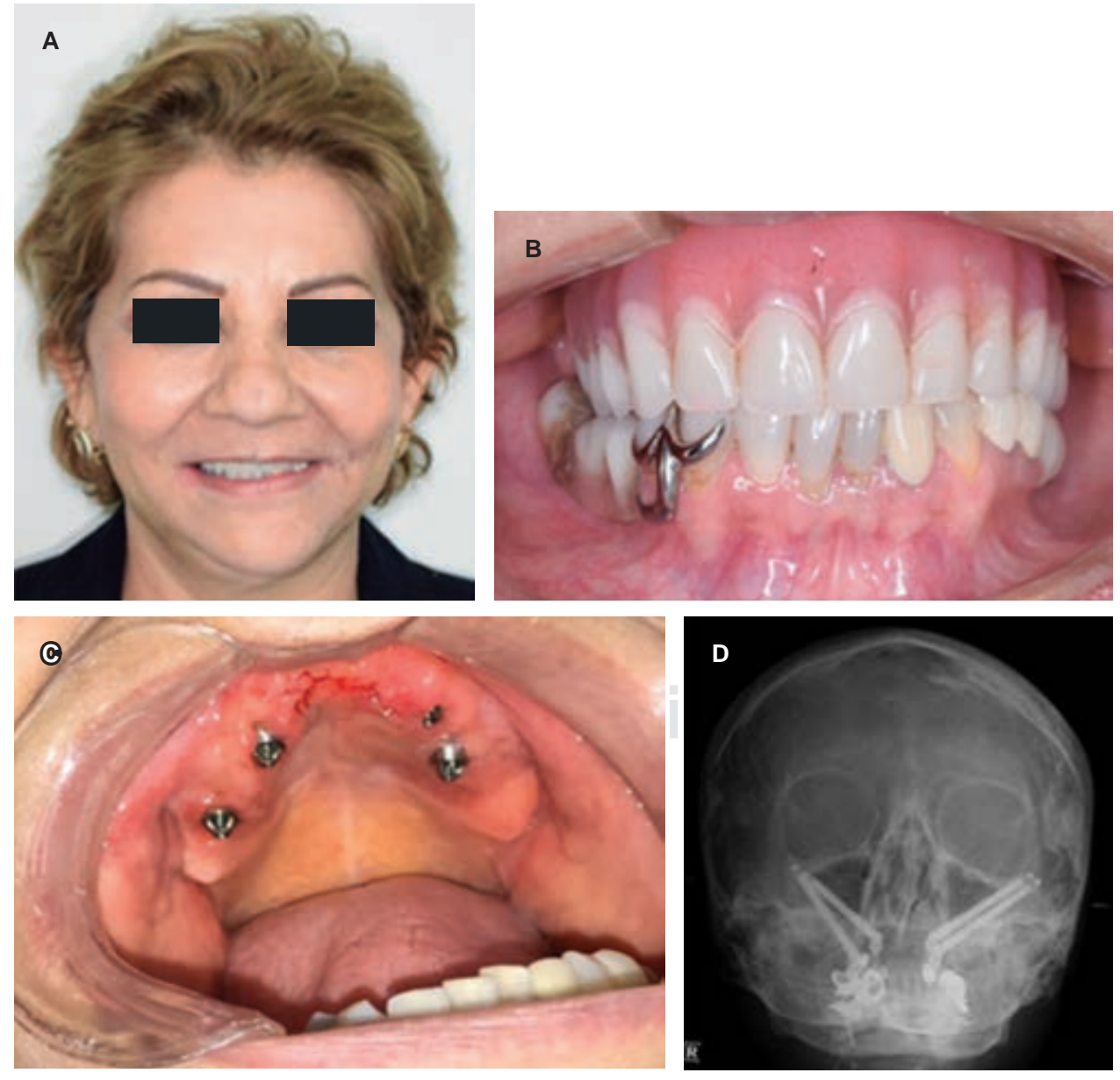

Figura 7:

A) Foto facial, B) prótesis provisional, C) aditamentos protésicos, D) radiografía de Waters de control. 
irrigación con S.S. $0.9 \%$ se realiza una ventana ósea a nivel de la cara anterior maxilar de $10 \times$ $10 \mathrm{~mm}$ aproximadamente, después se diseca la membrana sinusal hasta separarla en su totalidad de la cara lateral antral. A continuación, se coloca guía quirúrgica para determinar localización ideal del implante y se aplica el protocolo de fresado según la técnica original descrita por Branemark (Branemark System Zygoma Surgical Kit, Nobelbiocare, Zürich, Suiza). Se inicia la secuencia con fresa redonda continuando con la inicial 2.9 , luego la fresa de transición 2.9/3.5 y por último la 3.5, cada paso se realiza con abundante irrigación con S.S. $0.9 \%$. Una vez completada la preparación se midió la profundidad y se determinó la longitud del implante. Este mismo protocolo se realizó de manera bilateral insertándose cuatro implantes cigomáticos de $45 \mathrm{~mm}$ de longitud con un torque de inserción de $40 \mathrm{Ncm}$ cada uno. Se retiró el aditamento de transferencia y se colocaron los pilares angulados de Branemark multi-unit verificando su paralelismo. Se irriga profusamente con S.S. $0.9 \%$, control de hemostasia y se realiza síntesis de herida quirúrgica con sutura no reabsorbible de PTFE 4-0. Una vez suturadas las heridas la conexión de los pilares fue cargada de forma inmediata con una prótesis provisional de acrílico. Se terminó el acto quirúrgico sin incidentes ni accidentes, la paciente egresó de sala de operaciones despierta con su prótesis estable y funcional (Figura 4). Se estableció manejo médico a base de analgésicos/antiinflamatorios y antibióticos así como medidas de higiene oral y cuidados postoperatorios. La paciente se presentó a control postoperatorio a los 10 días con sus heridas quirúrgicas sin ningún dato patológico y prótesis provisional estable. A tres meses de seguimiento la paciente se mantiene asintomática con los implantes y prótesis estables a la espera de la prótesis definitiva (Figuras 5 a 7 ).

\section{DISCUSIÓN}

La rehabilitación estética y funcional en pacientes con secuelas por traumatismos, por resección de neoplasias o malformaciones congénitas de la región maxilofacial, así como pacientes con maxilares atróficos por otras causas, es un reto para cirujanos maxilofaciales y rehabilitadores orales. Se han documentado diversas técnicas quirúrgicas (injertos microvasculares, injertos libres onlay/inlay, injerto con elevación sinusal, etc.), las cuales tienen como objetivo la rehabilitación de los pacientes con implantes convencionales. Se sabe que la tasa de fracaso aumenta con la reducción de la altura ósea residual y la reducción de la longitud del implante. ${ }^{20-22}$ La técnica de elevación de seno maxilar con injerto óseo por mucho tiempo fue la de elección para resolver diversos casos de atrofia maxilar posterior, la Academia de Osteointegración estableció en 1994 su documento de consenso referente a los procedimientos de elevación sinusal en el cual establecen, después de revisar los resultados de 2,997 implantes colocados en 1,007 senos injertados con un seguimiento mínimo de tres años posterior a la rehabilitación, que la tasa de fracaso de este procedimiento con implante inmediato es de $61 \% .{ }^{23}$ Si bien el uso de injertos óseos autólogos es el «estándar de oro» de la regeneración, su utilización en combinación con implantes inmediatos o retardados tiene una tasa de fracaso de $10-30 \%$. A pesar de las numerosas publicaciones, la efectividad de los procedimientos de injerto sinusal sigue siendo controvertida. ${ }^{24-27}$

Se han planteado múltiples alternativas a los procedimientos de injerto óseo. La utilización de la sutura pterigomaxilar ha sido identificada como un sitio alternativo para la colocación de implantes. ${ }^{28-30}$ Otros autores han sugerido el uso de implantes inclinados y/o cortos para evitar la necesidad de procedimientos de elevación del seno. En los casos de cantidad de hueso adecuado en las zonas 1 y 2, según Bedrossian, se ha propuesto el uso de cuatro a seis implantes convencionales, inclinando el más distal de cada lado para lograr una buena distribución de la carga y así evitar la necesidad de injerto óseo. . $^{3-35}$

Durante las dos últimas décadas se han realizado numerosos estudios que demuestran que los implantes cigomáticos son una opción efectiva en el manejo del maxilar atrófico así como en defectos de postmaxilectomía, pues reducen la morbilidad quirúrgica y simplifican el tratamiento de rehabilitación en este tipo de pacientes. ${ }^{1-3} \mathrm{El}$ uso de múltiples implantes cigomáticos (2-3 por lado) para soportar una prótesis fue sugerido por Bothur y colaboradores. ${ }^{36}$ Los pacientes con atrofia maxilar severa (zonas 1, 2 y 3 según Bedrossian) son candidatos para el uso de implantes cigomáticos bilaterales con rehabilitación inmediata. En pacientes con atrofia maxilar no oncológica, la trayectoria de uno o ambos implantes cigomáticos puede situarse fuera del seno maxilar. ${ }^{37,38} \mathrm{La}$ 
Tabla 4: Clasificación de Lund-Mackay.

\begin{tabular}{|c|c|c|c|c|}
\hline Región & Lado & Normal & $\begin{array}{l}\text { Opaci- } \\
\text { ficación } \\
\text { parcial }\end{array}$ & $\begin{array}{l}\text { Opaci- } \\
\text { ficación } \\
\text { total }\end{array}$ \\
\hline Etmoidal & Derecho & 0 & 1 & 2 \\
\hline Anterior & Izquierdo & 0 & 1 & 2 \\
\hline Etmoidal & Derecho & 0 & 1 & 2 \\
\hline Posterior & Izquierdo & 0 & 1 & 2 \\
\hline \multirow[t]{2}{*}{ Maxilar } & Derecho & 0 & 1 & 2 \\
\hline & Izquierdo & 0 & 1 & 2 \\
\hline \multirow[t]{2}{*}{ Frontal } & Derecho & 0 & 1 & 2 \\
\hline & Izquierdo & 0 & 1 & 2 \\
\hline \multirow[t]{3}{*}{ Esfenoidal } & Derecho & 0 & 1 & 2 \\
\hline & Izquierdo & 0 & 1 & 2 \\
\hline & & $\begin{array}{c}\text { No } \\
\text { obstruido }\end{array}$ & & Obstruido \\
\hline Complejo & Derecho & 0 & & 2 \\
\hline $\begin{array}{c}\text { Osteo-meatal } \\
\text { Total }\end{array}$ & Izquierdo & 0 & & 2 \\
\hline
\end{tabular}

tasa de supervivencia reportada por Davo y su equipo ${ }^{39}$ en 36 implantes cigomáticos cargados inmediatamente fue de $100 \%$ con un seguimiento de seis a 29 meses, mientras que con los implantes convencionales se perdieron tres de 68 en el mismo tiempo de seguimiento. Balshi y colegas ${ }^{40}$ en un análisis retrospectivo de 56 pacientes que recibieron 110 implantes cigomáticos con carga inmediata reportaron un rango de supervivencia de $96 \%$ en un periodo de control de cinco años. Branemark y colaboradores ${ }^{41}$ en un estudio inicial informaron de 27 pacientes con defectos maxilares tratados con 65 implantes cigomáticos sin ninguna pérdida en un seguimiento de uno a 12 años. En otro estudio este mismo autor reportó una tasa de supervivencia de $94 \%$ en 28 pacientes edéntulos con maxilares severamente reabsorbidos tratados con 52 implantes cigomáticos con un seguimiento de cinco a 10 años. ${ }^{7}$ Otros autores como Kahnberg y su equipo ${ }^{42}$ reportaron en un estudio prospectivo multicéntrico una tasa de supervivencia de los implantes cigomáticos de $96.3 \%$ con un seguimiento de tres años. Malevez y colegas ${ }^{43}$ encontraron retrospectivamente una tasa de supervivencia de $100 \%$ para 103 implantes en 55 pacientes después de seis a 48 meses de carga. Fernández y colaboradores ${ }^{44}$ presentan un estudio retrospectivo de 80 pacientes con atrofia maxilar severa, a quienes se les colocaron 244 implantes cigomáticos con un éxito de $99.6 \%$ a 48 meses de seguimiento, este estudio representa la serie más grande de implantes cigomáticos publicada en Latinoamérica. En una revisión de 32 estudios clínicos de implantes cigomáticos realizada por Aparicio y su equipo ${ }^{45}$ se incluyeron las publicaciones de 1,031 pacientes con 2,131 implantes cigomáticos con un periodo de seguimiento de seis meses a 12 años, en total fracasaron 42 implantes dando una tasa de supervivencia global de $98.1 \%$. La salud del seno maxilar no parece verse comprometida como lo reporta Petrusson, ${ }^{46}$ quien realizó rinoscopia y sinuscopia a 14 pacientes que fueron tratados con implantes cigomáticos de 16 a 64 meses postoperatorios y no encontró signos de inflamación sinusal alrededor de los implantes. Davó y colegas ${ }^{47}$ evaluaron los senos maxilares mediante TAC preoperatoria y postoperatoria en 26 pacientes a quienes se les colocaron 52 implantes cigomáticos con carga inmediata con un seguimiento promedio de 21.9 meses, estos autores encontraron que entre 15 y $20 \%$ de los pacientes tenían engrosamiento asintomático de la mucosa sinusal.

Los datos preliminares de todos estos estudios muestran que el implante cigomático es altamente predecible, presentando buenos resultados clínicos a largo plazo y los hallazgos muestran que la carga inmediata es una modalidad de tratamiento viable cuando se incluyen implantes cigomáticos en el tratamiento.

Aparicio y colaboradores ${ }^{10}$ proponen «el código del éxito cigomático», una serie de criterios para describir el éxito/supervivencia de los implantes cigomáticos, el cual detalla los criterios para el éxito de una rehabilitación anclada en implantes cigomáticos. Estos criterios son distintos a los aplicados a la rehabilitación con implantes convencionales.

Tabla 5: Factores asociados con el diagnóstico de rinosinusitis.

\begin{tabular}{ll}
\hline Criterios mayores & Criterios menores \\
\hline $\begin{array}{l}\text { Presión o dolor facial } \\
\text { Congestión facial o } \\
\text { plenitud }\end{array}$ & $\begin{array}{l}\text { Dolor de cabeza } \\
\text { Obstrucción nasal }\end{array}$ \\
$\begin{array}{l}\text { Descarga purulenta } \\
\text { Hiposmia o anosmia }\end{array}$ & Halitosis \\
Pus en el examen nasal & Fatiga \\
Fiebre (sólo aguda) & Dolor dental \\
\hline
\end{tabular}


Tabla 6: Evaluación del éxito de los implantes cigomáticos.

\begin{tabular}{|c|c|c|c|c|}
\hline & \multicolumn{3}{|c|}{ Condición y grado de éxito } & \multirow{2}{*}{$\frac{\text { Fracaso, condición }}{\text { IV }}$} \\
\hline & I & II & III & \\
\hline $\begin{array}{l}\text { Criterio A } \\
\text { Estabilidad }\end{array}$ & $\begin{array}{l}\text { Sin movilidad } \\
\text { Sin dolor }\end{array}$ & $\begin{array}{l}\text { Ligera movilidad } \\
\text { Sin dolor }\end{array}$ & $\begin{array}{c}\text { Movilidad } \\
\text { moderada } \\
\text { (sin evidencia } \\
\text { perdida de } \\
\text { integración apical) }\end{array}$ & $\begin{array}{l}\text { Movilidad severa } \\
\text { (perdida de } \\
\text { integración) } \\
\text { Rotación y dolor }\end{array}$ \\
\hline $\begin{array}{l}\text { Criterio B } \\
\text { Patología sinusal } \\
\text { asociada }\end{array}$ & $\begin{array}{l}\text { Lanza-Kennedy (-) } \\
\text { Lund-Mackay (0) }\end{array}$ & $\begin{array}{l}\text { Lanza-Kennedy (+) } \\
\text { Lund-Mackay (0) }\end{array}$ & $\begin{array}{l}\text { Lanza-Kennedy (-) } \\
\text { Lund-Mackay }(>0)\end{array}$ & $\begin{array}{l}\text { Lanza-Kennedy (+) } \\
\text { Lund-Mackay (> 0) }\end{array}$ \\
\hline $\begin{array}{l}\text { Criterio C } \\
\text { Condición del } \\
\text { tejido blando } \\
\text { periimplantar }\end{array}$ & Sin recesión & $\begin{array}{c}\text { Ligera recesión } \\
\text { (Cabeza del implante } \\
\text { expuesta sin cuerdas } \\
\text { expuestas) }\end{array}$ & $\begin{array}{c}\text { Recesión moderada } \\
\text { (exposición de hasta } 7 \\
\text { cuerdas) }\end{array}$ & $\begin{array}{c}\text { Recesión severa } \\
\text { (exposición mayor a } 7 \\
\text { cuerdas) }\end{array}$ \\
\hline $\begin{array}{l}\text { Criterio D } \\
\text { Condición } \\
\text { protésica }\end{array}$ & $\begin{array}{l}0 \mathrm{~mm}<\mathrm{D}<6 \mathrm{~mm} \\
-3 \mathrm{~mm}<\mathrm{D}<0 \mathrm{~mm}\end{array}$ & $\begin{array}{l}6 \mathrm{~mm}<\mathrm{D}<10 \mathrm{~mm} \\
-4 \mathrm{~mm}<\mathrm{D}<-3 \mathrm{~mm}\end{array}$ & $\begin{array}{l}10 \mathrm{~mm}<\mathrm{D}<15 \mathrm{~mm} \\
-5 \mathrm{~mm}<\mathrm{D}<-4 \mathrm{~mm}\end{array}$ & $\begin{array}{l}\mathrm{D}<15 \mathrm{~mm} \\
\mathrm{D}<-5 \mathrm{~mm}\end{array}$ \\
\hline
\end{tabular}

El código del éxito cigomático de un implante específico está representado por el resultado de las siguientes variables.

Estabilidad del implante cigomático (probado individualmente), patología sinusal asociada (valorada por medio de las escalas Lund-Mackay ${ }^{48}$ y Lanza-Kennedy ${ }^{49}$ ) (Tablas 4 y 5), estado de los tejidos blandos periimplantarios y la evaluación del éxito protésico basado en el posicionamiento final del implante cigomático con respecto al centro de la cresta alveolar en la dimensión horizontal (valora la posición de la cabeza del implante con el reborde alveolar residual, valores positivos indican implantes colocados de manera palatina, valores negativos indican implantes colocados vestibularmente) ${ }^{10}$ (Tabla 6).

\section{CONCLUSIÓN}

Los implantes cigomáticos con carga inmediata son un recurso que proporciona una solución predecible y rápida en una sola etapa al problema de déficit óseo como resultado de atrofia alveolar maxilar o maxilectomía. Son una opción de rehabilitación que evita meses de espera para la consolidación de un injerto óseo de elevación de seno maxilar, seguido del tiempo necesario para la integración del implante antes de la restauración. Un paciente de maxilectomía puede experimentar un plan de tratamiento muy acelerado y simplificado que permitirá la construcción de la prótesis definitiva en el menor tiempo posible después de la resección del tumor. Tanto en los grupos de pacientes atróficos como en los de maxilectomía, el implante cigomático no conlleva mayor complejidad protésica para el paciente y ha mostrado resultados clínicos superiores en comparación con el injerto óseo, por lo que representan un nuevo «estándar de oro» en cuanto al tratamiento de los huesos maxilares comprometidos (Tabla 6).

\section{REFERENCIAS}

1. Branemark PI, Higuchi KW, Oliveira MF. Rehabilitation of complex cleft palate and craniomaxillofacial defects: the challenge of Bauru. $1^{\text {st }}$ ed. Quintessence, 1999.

2. Higuchi KW. The zygomaticus fixture: an alternative approach for implant anchorage in the posterior maxilla. Ann R Australas Coll Dent Surg. 2000; 15: 28-33.

3. Malevez C, Daelemans P, Adrianssens P, Durdu F. Use of zygomatic implants to deal with resorbed posterior maxillae. Periodontol 2000. 2003; 33: 82-89.

4. Rigolizzo M, Camilli J, Francischone $\mathrm{C}$ et al. Zygomatic bone: anatomic basis for osseointegrated implant anchorage. Int J Oral Maxillofac Implants. 2005; 20: 441-447.

5. Nkenke E, Hahn M, Lell M, Wiltfang J, Schultze-Mosgau S, Stech $B$ et al. Anatomic site evaluation of the zygomatic bone for dental implant placement. Clin Oral Implants Res. 2003; 14: $72-79$.

6. Kato $\mathrm{Y}, \mathrm{Kizu} \mathrm{Y}$, Tonogi $\mathrm{M}$ et al. Internal structure of zygomatic bone related to zygomatic fixture. J Oral Maxillofac Surg. 2005; 63: 13251329.

7. Branemark PI, Grondahl K, Ohrnell LO et al. Zygoma fixture in the management of advanced atrophy of the maxilla: technique and 
long-term results. Scand J Plast Reconstr Surg Hand Surg. 2004; 38: $70-85$

8. Uchida Y, Goto M, Katsuki T et al. Measurement of the maxilla and zygoma as an aid in installing zygomatic implants. J Oral Maxillofac Surg. 2001; 59: 1193-1198.

9. Grondahl HG, Grondahl K, Lindfors N. Pre- and postoperative radiographic examinations of the zygoma implant site. In: Aparicio $\mathrm{C}$, editor. The anatomy guided approach. Berlin: Ed. Quintessence, 2012, 47-56.

10. Aparicio C, Manresa C, Francisco K et al. Zygomatic implants: indications, techniques and outcomes, and the zygomatic success code. Periodontol 2000. 2014; 66: 41-58.

11. Davo R, Malevez C, Rojas J, Rodríguez J, Regolf J. Clinical outcome of 42 patients treated with 81 immediately loaded zygomatic implants: a 12-to-42 month retrospective study. Eur J Oral Implantol. 2008; 1: 141-150.

12. Duarte LR, Filho HN, Francischone CE, Peredo LG, Braanemark PI. The establishment of a protocol for the total rehabilitation of atrophic maxillae employing four zygomatic fixtures in an immediate loading system - a 30-month clinical and radiographic follow-up. Clin Implant Dent Relat Res. 2007; 9: 186-196.

13. Bedrossian E, Sullivan RM, Fortin $Y$ et al. Fixed-prosthetic implant restoration of the edentulous maxilla: a systematic pretreatment evaluation method. J Oral Maxillofac Surg. 2008; 66: 112-122.

14. Stella J, Warner M. Sinus slot technique for simplification and improved orientation of zygomaticus dental implants: a technical note. Int J Oral Maxillofac Implants. 2000; 15: 889.

15. Boyes-Varley JG, Howes DG, Lownie JF et al. Surgical modifications to the Branemark zygomaticus protocol in the treatment of the severely resorbed maxilla: a clinical report. Int $J$ Oral Maxillofac Implants. 2003; 18: 232-237

16. Chow J, Hui E, Lee PK, Li W. Zygomatic implants-protocol for immediate occlusal loading: a preliminary report. Journal of Oral and Maxillofacial Surgery. 2006; 64 (5): 804-811.

17. Al-Nawas B, Wegener J, Bender C, Wagner W. Critical soft tissue parameters of the zygomatic implant. J Clin Periodontol. 2004; 31 : 497-500.

18. Aparicio C. A proposed classification for zygomatic implant patient based on the zygoma anatomy guided approach (ZAGA): a cross-sectional survey. Eur J Oral Implantol. 2011; 4: $269-275$.

19. Edward B. Sevetz Jr. Treatment of the severely atrophic fully edentulous maxilla: the zygoma implant option. Atlas Oral Maxillofacial Surg Clin N Am. 2006; 14: 121-136.

20. Rosen PS, Summers R, Mellado JR, Salkin LM, Shanaman RH, Marks MH, Fugazzotto PA. The bone-added osteotome sinus floor elevation technique: multicenter retrospective report of consecutively treated patients. Int J Oral Maxillofac Implants. 1999; 14: 853-858.

21. Widmark G, Andersson B, Carlsson GE, Ivanoff CJ, Lindvall AM. Rehabilitation of patients with severely resorbed maxillae by means of implants with or without bone grafts: a 1 year follow up study. Int J Oral Maxillofac Implants. 1998; 13: 474-482.

22. Collins TA, Brown GK, Johnson N, Massey JA, Nunn BD. Team management of atrophic edentulous with autogenous inlay, veneer, and split grafts and endosseous implants: case reports. Quintessence Int. 1995: 26: 79-93.

23. Shulman LB, Jensen OT. Academy of osseointegration, sinus graft consensus conference. Int $\mathrm{J}$ Oral Maxillofac Implants. 1998; $13: 4$.

24. Keller EE. Reconstruction of the severely atrophic edentulous mandible with endosseous implants: a 10 year longitudinal study. $J$ Oral Maxillofac Surg. 1995: 53: 317-318.
25. Lekholm U, Zarb GA. Patient selection and preparation. In:Branemark P-I, Zarb GA, Albrektsson T, editors. Tissue integrated prostheses: osseointegration in clinical dentistry. Chicago, IL: Quintessence, 1985, 199-209.

26. Sjostrom M, Sennerby L, Nilson H, Lundgren S. Reconstruction of the atrophic edentulous maxilla with free iliac crest grafts and implants: a 3-year report of a prospective clinical study. Clin Implant Dent Relat Res. 2007; 9: 46-59.

27. Graziani F, Donos N, Needleman I et al. Comparison of implant survival following sinus floor augmentation procedures with implants placed into pristine posterior maxillary bone: a systematic review. Clin Oral Implants Res. 2004; 15: 677-682.

28. Fernandez VJ, Fernández VJ. Placement of screw type implants in the pterigomaxillary pyramidal region: surgical procedure and preliminary results. Int J Oral Maxillofac Implants. 1997; 12: 814-819.

29. Graves SL. The pterigoid plate implant: a solution for restoring the posterior maxilla. Int J Periodontics Restorative Dent. 1994; 14 : 513-523.

30. Tulasne JF. Osseointegrated fixtures in the pterygoid region. In: Worthington P, Branemark PL, editors. An advanced osseointegration surgery: applications in the maxillofacial region. Chicago, IL: Quintessence, 1992, 182-188.

31. Malo P, Rangert B, Nobre M. All-on-4 immediate-function concept with Branemark System implants for completely edentulous maxillae: a 1-year retrospective clinical study. Clin Implant Dent Relat Res. 2005; 7: S88-S94

32. Aparicio C, Perales P, Rangert B. Tilted implants as an alternative to maxillary sinus grafting: a clinical, radiologic, and periotest study. Clin Implant Dent Relat Res. 2001; 3: 39-49.

33. Krekmanov L, Kahn M, Rangert B et al. Tilting of posterior mandibular and maxillary implants for improved prosthesis support. Int J Oral Maxillofac Implants. 2000; 15: 405-414.

34. Zampelis A, Rangert B, Heijl L. Tilting of splinted implants for improved prosthodontic support: a two-dimensional finite element analysis. J Prosthet Dent. 2007; 97: S35-S43.

35. Calandriello R, Tomatis M. Simplified treatment of the atrophic posterior maxilla via immediate/early function and tilted implants: a prospective 1-year clinical study. Clin Implant Dent Relat Res. 2005; 7: S1-S12.

36. Bothur S, Jonsson G, Sandahl L. Modified technique using multiple zygomatic implants in reconstruction of the atrophic maxilla: a technical note. Int J Oral Maxillofac Implants. 2003; 18: 902-904.

37. Schmidt BL, Pogrel MA, Young CW et al. Reconstruction of extensive maxillary defects using zygomaticus implants. J Oral Maxillofac Surg. 2004; 62: 82-89.

38. Maló P, Nobre M de A, Petersson $U$ et al. A pilot study of complete edentulous rehabilitation with immediate function using a new implant design: case series. Clin Implant Dent Relat Res. 2006; 8: 223-232.

39. Davo C, Malevez C, Rojas J. Immediate function in the atrophic maxilla using zygoma implants: a preliminary study. J Prosthet Dent. 2007; 97: S44-S51.

40. Balshi SF, Wolfinger GJ, Balshi TJ. A retrospective analysis of 110 zygomatic implants in a single-stage immediate loading protocol. Int J Oral Maxillofac Implants. 2009; 24 (2): 335-41.

41. Branemark PI, Groondahl K, Worthington P. Appraisal of restored anatomy and function. In: Branemark PI, Groondahl K, Worthington $\mathrm{P}$, editors. Osseointegration and autogenous bone grafts: reconstruction of the edentulous atrophic maxilla. Berlin: Ed. Quintessence, 2001, 1-13.

42. Kahnberg KE, Henry P, Hirsch J-M et al. Clinical evaluation of the zygoma implant: 3-year follow-up at 16 clinics. J Oral Maxillofac Surg. 2007; 65: 2033-2038. 
Rev Mex Cir Bucal Maxilofac. 2021;17 (2): 96-108

43. Malevez C, Abarca M, Durdu F et al. Clinical outcome of 103 consecutive implants: a 6-48 month follow-up study. Clin Oral Implants Res. 2004; 15: 18-22.

44. Fernández H, Gómez-Delgado A, Trujillo-Saldarriaga $S$ et al. Zygomatic implants for the management of the severely atrophied maxilla: a retrospective analysis of 244 implants. J Oral Maxillofac Surg. 2014; 72 (5): 887-891.

45. Aparicio C, Ouazzani W, Hatano N. The use of zygomatic implants for prosthetic rehabilitation of the severely resorbed maxilla. Periodontol 2000. 2008; 47: 162-171.
46. Petruson B. Sinuscopy in patients with titanium implants in the nose and sinuses. Scand J Plast Reconstr Surg Hand Surg. 2004; 38: 86-93.

47. Davó R, Malevez C, López-Orellana C et al. Sinus reactions to immediately loaded zygoma implants, a clinical and radiographic study. Eur J Oral Implantol. 2008; 1: 53-60.

48. Lund VJ, Mackay IS. Staging in rhinosinusitis. Rhinology. 1993; 31: 183-184.

49. Lanza DC, Kennedy DW. Adult rhinosinusitis defined. Otolaryngol Head Neck Surg. 1997: 117: s1-s7. 